\title{
Aproximación al manejo de la psoriasis en el pie
}

\author{
An approach to the management of psoriasis in the foot
}

\section{Daniel López López ${ }^{1}$, David Rodríguez Sanz ${ }^{2}$, Alfredo Soriano Medrano ${ }^{3}$, Noemia Zeltia López MARTínez ${ }^{4}$}

\author{
${ }^{1}$ Doctor por la Universidade da Coruña. Profesor Colaborador. Departamento de Ciencias da Saúde. Facultad de \\ Enfermería e Podoloxía. Universidade da Coruña. \\ daniellopez@udc.es \\ ${ }^{2}$ Doctor por la Universidad Rey Juan Carlos. Profesor Universidad Europea de Madrid. \\ davidrodriguezsanz@hotmail.com \\ ${ }^{3}$ Doctor por la Universidad Rey Juan Carlos. Clinisalud Centro Médico, Albacete, España. \\ soriano.alfredo@gmail.com \\ ${ }^{4}$ Experto en Podología Médico Quirúrgica del Antepie por la Universidad Complutense de Madrid. Profesor Aso- \\ ciado. Departamento de Ciencias da Saúde. Facultad de Enfermería e Podoloxía. Universidade da Coruña. \\ memitos34@hotmail.com
}

Correspondencia:

Dr Daniel López López

Universidade da Coruña

Profesor Colaborador

Departamento de Ciencias da Saúde

Facultad de Enfermaría e Podoloxía

Campus Universitario de Esteiro s/n

E-15403 Ferrol

Correo electrónico: daniellopez@udc.es

Fecha de recepción: 20 de mayo de 2013

Fecha de aceptación: 22 de julio de 2013

Los autores declaran no tener ningún tipo de interés económico o comercial.

\section{RESUMEN}

La psoriasis es una enfermedad cutánea, no contagiosa, de carácter inflamatorio, generalmente de curso crónico y recidivante. Afecta al 3\% de la población, siendo el origen idiopático, si bien parece estar relacionada con el sistema inmunitario y con factores ambientales en personas con una susceptibilidad genética. Las lesiones son usualmente distribuidas simétricamente y están característicamente localizadas a nivel general en las orejas, los codos, las rodillas, el ombligo, la hendidura glútea, los genitales y el cuero cabelludo y de manera especial en los pies y en las uñas. Esta afección ocasiona perturbación ocupacional, deterioro psicológico y físico, y una potencial estigmatización social.

La carencia de consenso en relación a la etiología multifactorial que presenta esta afección y la prevalencia de esta enfermedad a nivel del pie, nos lleva a mejorar los conocimientos existentes en lo que se refiere a su clasificación, diagnóstico y tratamiento en la búsqueda de actualizar los conceptos relacionados con esta enfermedad no transmisible. Palabras clave: psoriasis; placas psoriásicas; pie; guías clínicas; concepto.

\begin{abstract}
Psoriasis is a skin disease, not contagious, inflammatory in nature, generally chronic and relapsing course. It affects $3 \%$ of the population, being idiopathic, although it seems to be related gives the immune system and environmental factors in people with a genetic susceptibility. The lesions are usually symmetrically distributed and are typically located at a general level in the ears, elbows, knees, umbilicus, gluteal cleft, the genitals and the scalp and especially
\end{abstract}


in the feet and nails. This condition causes disturbance occupational psychological and physical deterioration, and potential social stigmatization.

The lack of consensus regarding the multifactorial etiology has this condition and the prevalence of this disease at foot level, leads to improved knowledge in regard to their classification, diagnosis and treatment in the quest to update concepts related to this non communicable disease.

Key words: psoriasis; psoriatic plaques; foot; clinical guidelines; concept.

Referencia normalizada: López López, D., Rodríguez Sanz, D., Soriano Medrano, A., López Martínez, N.Z. Aproximación al manejo de la psoriasis en el pie. Rev. Int. Cienc. Podol. 2014; 8(1): 9-24.

Sumario: 1. Introducción, 2. Fisiopatología, 3. Clínica, 4. Diagnóstico, 5. Tratamiento, 6. Conclusiones, Bibliografía. 


\section{INTRODUCCIÓN}

La psoriasis se define como un trastorno inmune sistémico mediado por procesos inflamatorios y caracterizado por la presencia de manifestaciones articulares y en la piel. Afecta al 3\% de la población occidental, con diferente prevalencia entre los diferentes grupos étnicos y con un impacto significativo en la calidad de vida $^{1,2}$

Se trata de una enfermedad crónica no contagiosa y recurrente de la piel, que puede aparecer a cualquier edad, aunque muchos estudios han demostrado que la edad de aparición de los brotes y picos de la enfermedad está relacionada con la adolescencia y también con la etapa adulta alrededor de los 50-60 años ${ }^{3,4}$.

La prevalencia mundial de esta dermatosis crónica es elevada y se asocia a la concomitancia con otras enfermedades crónicas inflamatorias, tales como la artritis reumatoidea y la enfermedad inflamatoria del intestino, que ocasionan una merma en la calidad de vida de las personas afectadas, perturbación ocupacional, deterioro psicológico, físico y una potencial estigmatización social ${ }^{5,6}$.

A nivel del pie, la gravedad de la enfermedad se produce independientemente del grado de participación del área de superficie corporal en la que se localice, a pesar de que esta zona anatómica representa solo el $2 \%$ de la superficie corporal la significativa morbilidad puede tener un efecto debilitante sobre las funciones diarias del paciente, siendo las quejas habituales en la consulta las que se relacionan con el deterioro de la movilidad, el dolor, la discapacidad, el prurito y la vergüenza ${ }^{7,8}$.

Además las personas con psoriasis severa tienen un mayor riesgo de presentar enfermedades cardiovasculares, diabetes, depresión, hipertensión, obesidad e hiperlipidemia ${ }^{9,10}$.

Los médicos, incluidos los dermatólogos, prefieren dejar de estimar la magnitud de la discapacidad y la satisfacción de sus pacientes, aunque la carga de la enfermedad puede producir morbilidad funcional, psicológica y social, incluso en personas con una de participación mínima $^{11}$.

Sin embargo, el manejo y el avance de las investigaciones relacionadas con la psoriasis se ha incrementado en los últimos 20-30 años, existiendo en la actualidad tratamientos eficaces que mejoran los resultados y contribuyen a la gestión de la psoriasis, incluyendo su impacto en el bienestar de los pacientes, los profesionales sanitarios y que viene apoyada por la necesidad de guías basadas en la evidencia para el tratamiento ${ }^{12,13}$.

\section{FISIOPATOLOGÍA}

La investigación reciente sugiere que la psoriasis es una enfermedad compleja, al igual que la comprensión y la percepción de la fisiopatología de la enfermedad que ha evolucionado desde un trastorno hiperqueratósico mediado por los queratinocitos a una disrregulación del sistema inmune mediado por las citoquinas, que según distintos estudios se observan cambios en la expresión de la enfermedad de la piel, a una enfermedad de las células T que demuestra el grado de compromiso sistémico, donde las colonias de las células T-helper (Th-1, Th-17 y Th-22) se estimulan en su diseminación para liberar la respuesta inflamatoria por medio de las citoquinas, incluyendo el factor de necrosis tumoral $-\alpha$ (TNF- $\alpha$ ) y la interleuquina (IL-17 y IL-22) $)^{14,15}$.

En el inicio de la enfermedad, así como durante los brotes que se ocasionan más tarde, las células dendríticas especiales en la epidermis y en la dermis se activan y producen las sustancias TNF- $\alpha$ e IL-23, que a su vez, promueven el desarrollo de ciertas subclases de células $\mathrm{T}$ (Th-1, Th-17). Estas células T segregan mediadores que contribuyen a la vascularización y a cambios en la epidermis ${ }^{16}$.

La activación de la señal intracelular en vías de transducción juega un papel esencial en el refuerzo la reacción inflamatoria inmune, al igual que los antimicrobianos péptidos (AMP) que son un componente importante del sistema inmune innato y desempeñan un papel importante en la homeostasis de órganos superficiales tales como la piel ${ }^{16,17}$ (véase Figura 1).

Además todos los amplificadores que han sido identificadas hasta la fecha están presentes en cantidades aumentadas en lesiones de psoriasis, la sobreexpresión de los péptidos antimicrobianos es característico de la psoriasis y puede ser interpretado como una señal de acti- 


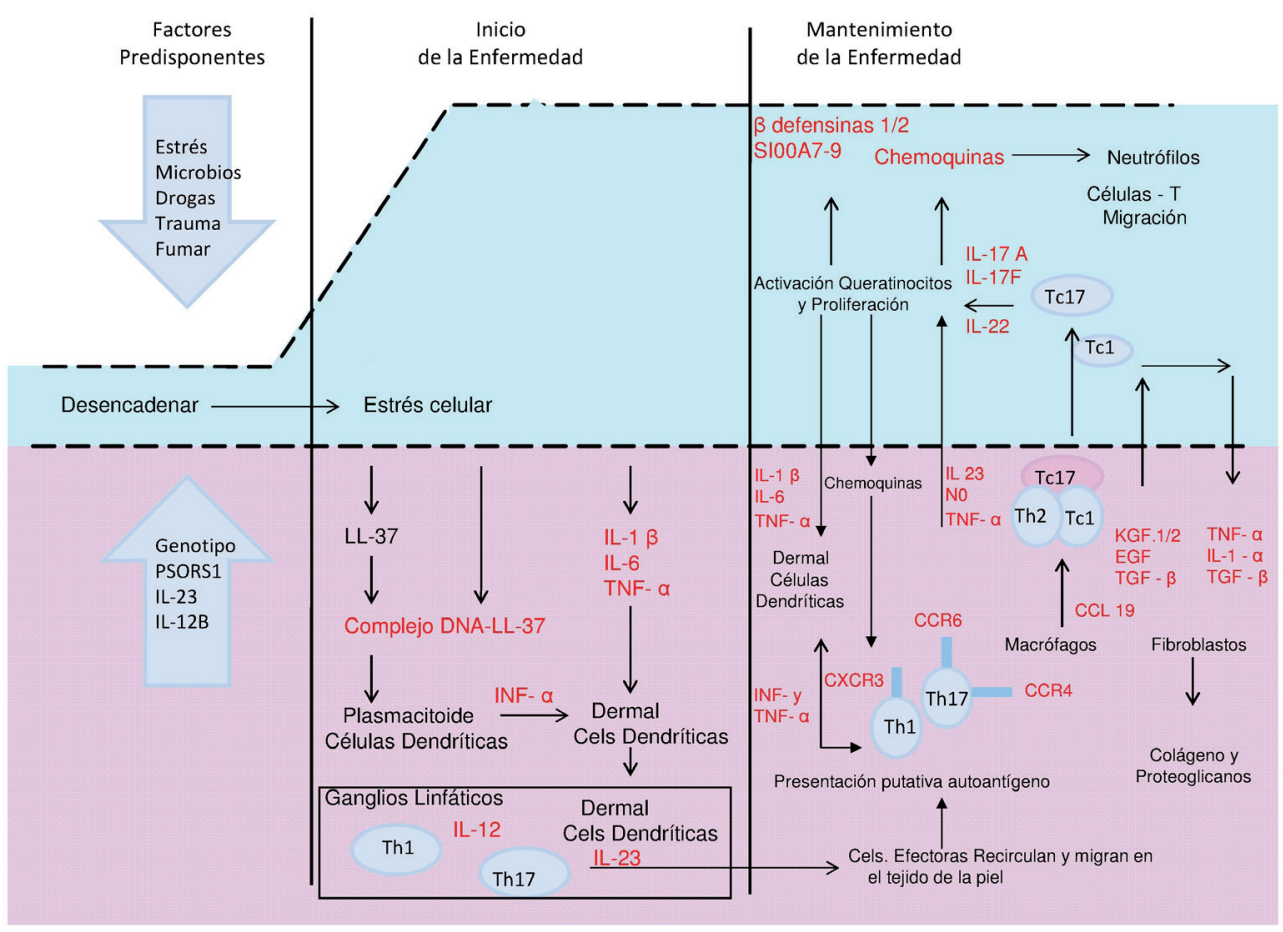

Figura 1. Esquema de inicio y evolución de una lesión psoriásica. La línea punteada representa la barrera de la piel, que se dilata con los procesos patológicos que la activan. Los moduladores inmunes, aparecen marcados de color rojo e incluyen las citoquinas y en color azul las células defensivas. Los procesos se indican con un recuadro (adaptado de Nestle, Kaplan, Barker, 2009; Reich, 2012).

vación del sistema inmune innato. De acuerdo con esta hipótesis, la infección bacteriana de las lesiones psoriásicas no es observada en la práctica clínica, en contraste con la de la dermatitis atópica que si aparece afectada ${ }^{18}$.

Además, se han implicado varios factores exógenos y endógenos que exacerban la enfermedad, incluidos el etanol, el estrés, las infecciones, las quemaduras solares, el trauma local, los medicamentos, el tabaquismo, etc ${ }^{16}$.

\section{CLÍNICA}

La rápida renovación de las células de la piel en las personas con psoriasis, cada 3-5 días, en comparación a las personas sin psoriasis cada 28-30 días, es lo que permite un acúmulo y un exceso de la cantidad de células en la superficie cutánea y contribuye a la aparición de las diferentes clases de lesiones y de formas de presentación de esta enfermedad, que pueden aparecer de forma aislada, simultáneamente o secuencialmente ${ }^{19}$.

Las manifestaciones clínicas de la psoriasis son bien conocidas y por lo general se reconocen fácilmente, aunque la presentación y la ubicación de la psoriasis puede variar en las diferentes etapas de la enfermedad (véase Tabla $1)^{20}$.

Según el Consenso Internacional de la Psoriasis la clasificación para su uso clínico y con fines de investigación está relacionada con el fenotipo de la enfermedad y así se pueden diferenciar entre otros ${ }^{21}$ :

1. Psoriasis en Placa o Vulgar: es la forma usual de presentación y está presente en aproximadamente el $90 \%$ de los casos, caracterizado por un enrojecimiento, por la aparición de escamas, y de lesiones discoi- 


\begin{tabular}{cc} 
Localización & $\begin{array}{c}\text { \% de Psoriasis } \\
\text { en Pacientes }\end{array}$ \\
\hline Cuero cabelludo & 80 \\
Codos & 78 \\
\hline Piernas & 74 \\
\hline Rodillas & 57 \\
Brazos & 54 \\
\hline Tronco & 53 \\
\hline Parte inferior del & 47 \\
cuerpo & 12 \\
\hline Base de la espalda & 38 \\
\hline Otros & 38 \\
\hline Manos y Pies & \\
\hline
\end{tabular}

Tabla 1. Lesiones en pacientes con psoriasis, según Kerkhof, 2003.

des (placas) de al menos $0,5 \mathrm{~cm}$ de diámetro $^{1,22}$ (véase Figura 2).

Las placas puede ocurrir como lesiones únicas en sitios predispuestos (por ejemplo, las rodillas, los codos, las plantas de los pies) o en áreas amplias del cuerpo y también de manera generalizada. La brillante superficie de las placas puede mostrar agrietamiento secundario o maceración que permite observar la clara demarcación entre la placa y la piel normal circundante.

La localización de la psoriasis en placas lleva a que se clasifique de acuerdo a los sitios anatómicos específicos y a las variaciones fenotípicas, así podemos encontrar:

1.1. Psoriasis Flexural, Intertriginosa o Inversa: relacionada con escamas finas, placas de color rosado - rojizo, bien definidas sin apenas descamación (debido a la hume-

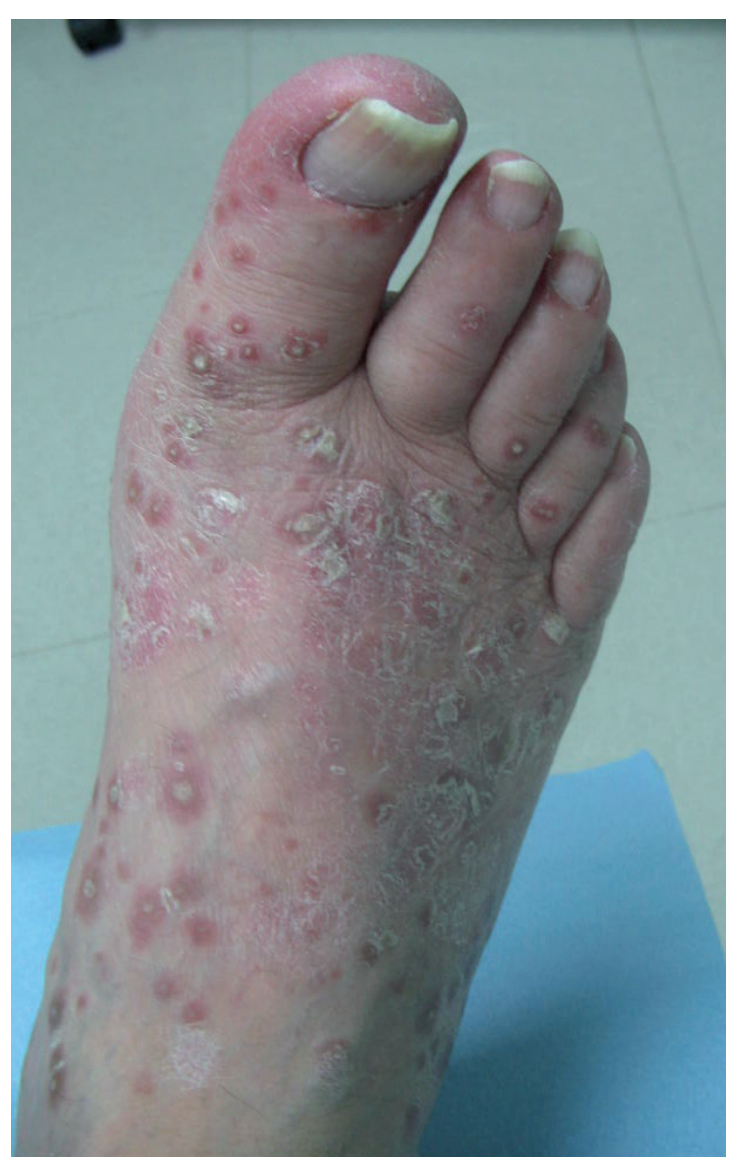

Figura 2. Psoriasis en Placa del pie derecho.

dad de la zona), que limitan a la piel en los pliegues como los de las zonas inframamaria, la ingle, la zona axilar, genital y / las regiones de la hendidura natal..., de forma bilateral y simétrica. La sintomatología suele establecerse con prurito y/o escozor y el tratamiento para obtener mejora clínica suele generar dificultad. La presencia de fricción, calor y la humedad inducen a la aparición de la psoriasis en los pliegues mediante un efecto Koebner. Además, también se ha sugerido la participación de algunas levaduras (Malassezia furfur) en su inducción ${ }^{23}$.

1.2. Psoriasis Ungueal: la afectación de la lámina ungueal está relacionada con los signos clínicos a los que esté asociado su localización anatómica (véase Tabla 2) y por lo general conduce al engrosamiento, piqueteado, decoloración, y a la fragmen- 
tación de la placa de la uña, así como a la separación de la lámina del lecho ungueal 24.

1.3. Psoriasis del Cuero Cabelludo: es la zona más habitual de presentación en las personas y rara vez se extiende más allá de $2 \mathrm{~cm}$ de la línea del cabello.

1.4. Psoriasis Palmoplantar (no pustulosa): la psoriasis en placa en las palmas de las manos o las plantas de los pies pueden tener una amplia gama de manifestaciones, caracterizado por el enrojecimiento confluente y el escalamiento sin placas discernibles o áreas mal delimitadas, escamosas o agrietadas en placas grandes que cubre la planta del pie o la palma de la mano y que se extiende a la piel circundante (véase Figura 3).

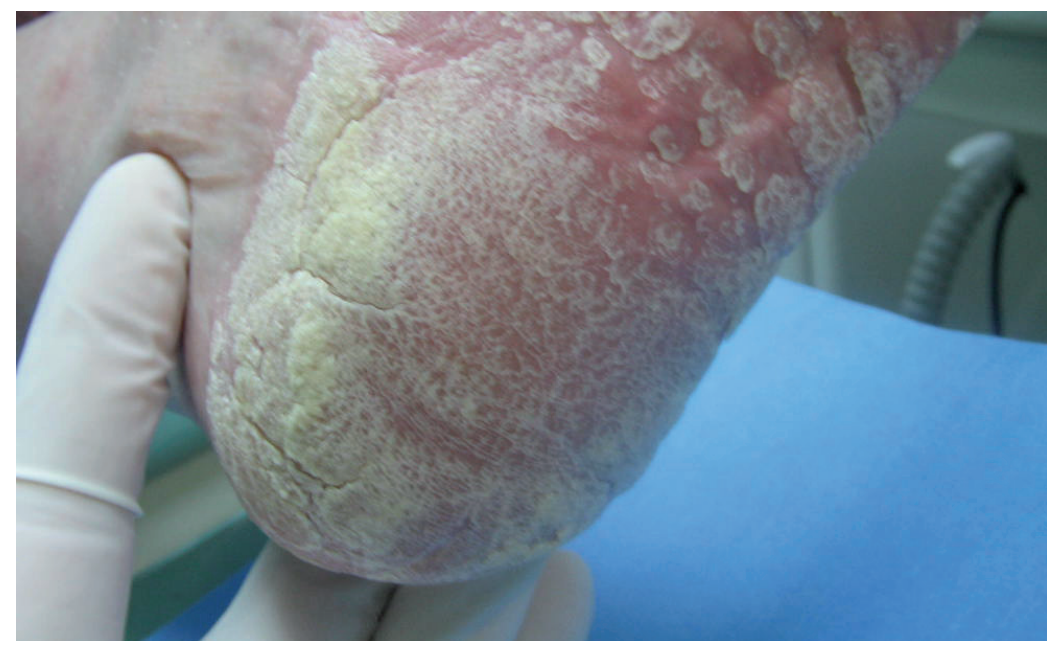

Figura 3. Psoriasis plantar no pustulosa en el pie izquierdo.
Matriz distal

Hiponiquio

Lecho ungueal

Lámina ungueal

Falanges de dedos

Pliegues ungueales

Matriz Proximal
Lámina ungueal fina, eritema de la lúnula

Hiperqueratosis subungueal, onicolisis.

Signo de "mancha de aceite",

Hiperqueratosis subungueal, onicolisis, hemorragias en astilla

Desmenuzamiento y destrucción y otras alteraciones secundarias en la zona específica

Artritis psoriásica con alteraciones

ungueales en más del $80 \%$ de los casos

Psoriasis Cutánea

Lineas de Beau, piqueteado, onicorrexis

Tabla 2. Localización y signos clínicos, según Padín, López, Martín y García, 2005. 
1.5. Sebopsoriasis: la forma seborreica de la psoriasis en placas tiene esa denominación como consecuencia de la similitud en la forma de presentación con la dermatitis seborreica que tiene una apariencia fina, roja, bien delimitada de las lesiones y que aparece de manera aislada o puede estar asociada con psoriasis en placa o en otras zonas corporales.

2. Psoriasis No en Placa: esta forma de psoriasis se presenta como una enfermedad aguda relacionada con la erupción de pequeñas pápulas en el tronco, las extremidades y los pies, las más habituales formas de presentación son:

2.1. Psoriasis en Gotas o Guttata: erupción espontánea de lesiones en forma de pequeñas gotas de color salmón, menores de 1 centímetro, a veces pruriginosa, que en alrededor de los dos tercios de los casos esta afección es provocada por una infección estreptocócica (véase Figura 4)

2.2. Psoriasis Pustulosa Generalizada o Psoriasis de Von Zumbusch: se caracteriza por pequeñas pústulas, monomórficas alojadas

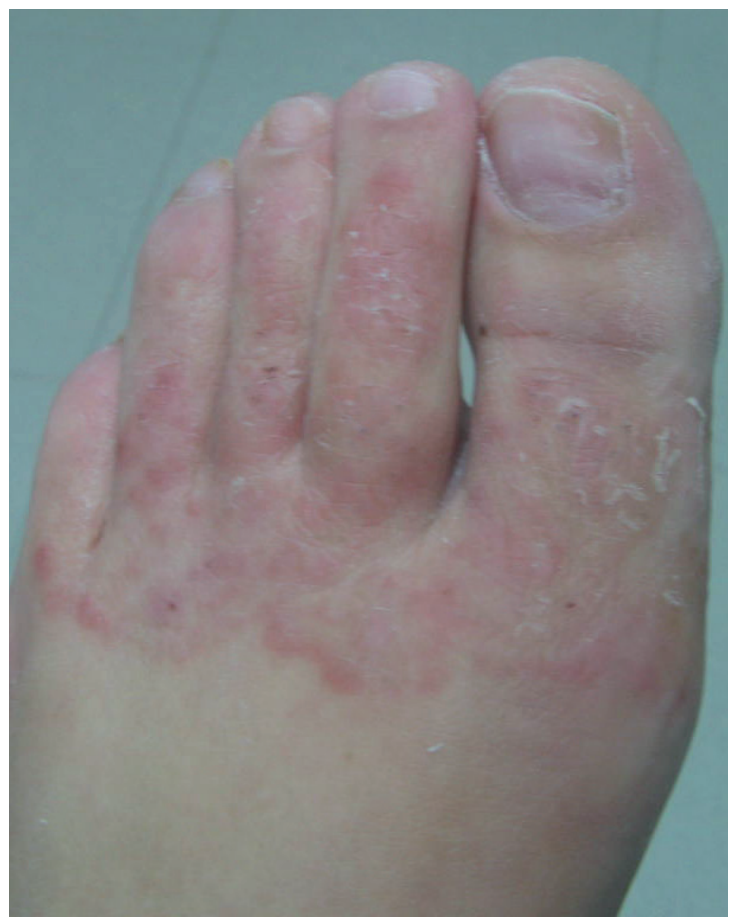

Figura 4. Psoriasis en Gotas en el pie izquierdo. en la piel eritrodérmica o a lo largo de los bordes de la expansión de placas inflamatorias. La aparición está acompañada de la psoriasis en placa o pueden presentar de novo. La variedad habitual es la psoriasis pustulosa palmoplantar en una quinta parte de los casos. (véase Figura 5).

2.3. Acrodermatitis continua de Hallopeau: abarca el área de las uñas y de la zona periungueal y se caracteriza por la presencia de pústulas, distrofia ungueal, enrojecimiento, descamación crónica periungueal e hinchazón. La enfermedad se asocia a menudo con psoriasis palmoplantar o en placas en otras áreas corporales.

2.4. Psoriasis Eritrodérmica o Exfoliativa: caracterizada por pérdida de la función y de la barrera de la piel que genera un compromiso en el control de la temperatura y del balance de líquidos y electrolitos, lo cual genera cambios hemodinámicos similares a los producidos por las quemaduras con enrojecimiento generalizado, prurito intenso, sensación de ardor y / o infecciones secundarias (véase Figura 6) ${ }^{25}$.

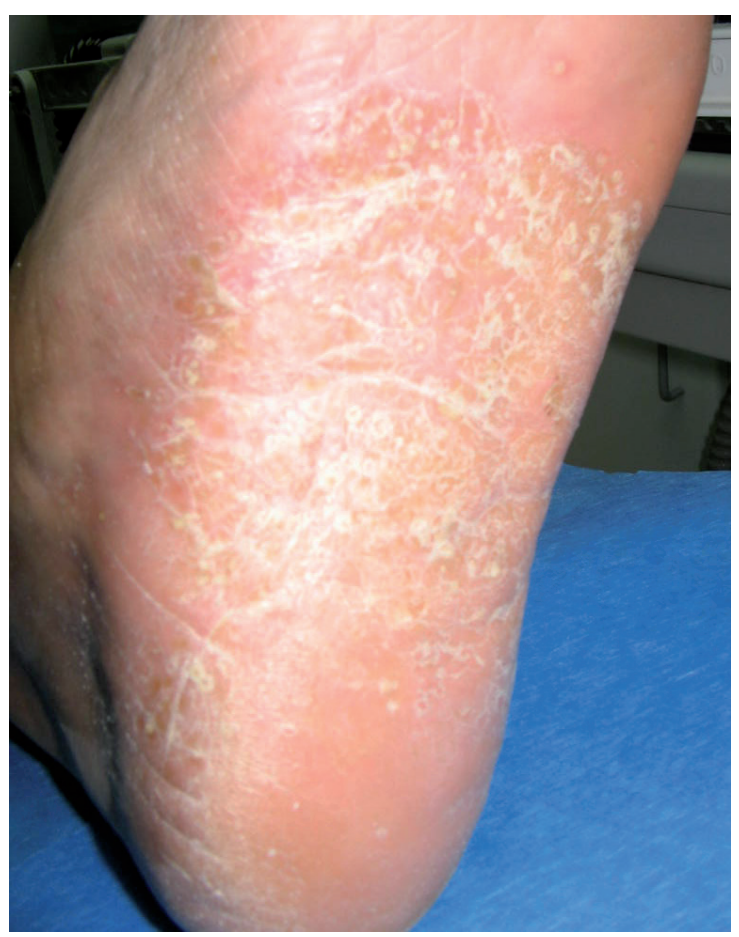

Figura 5. Psoriasis Pustulosa en el pie izquierdo. 


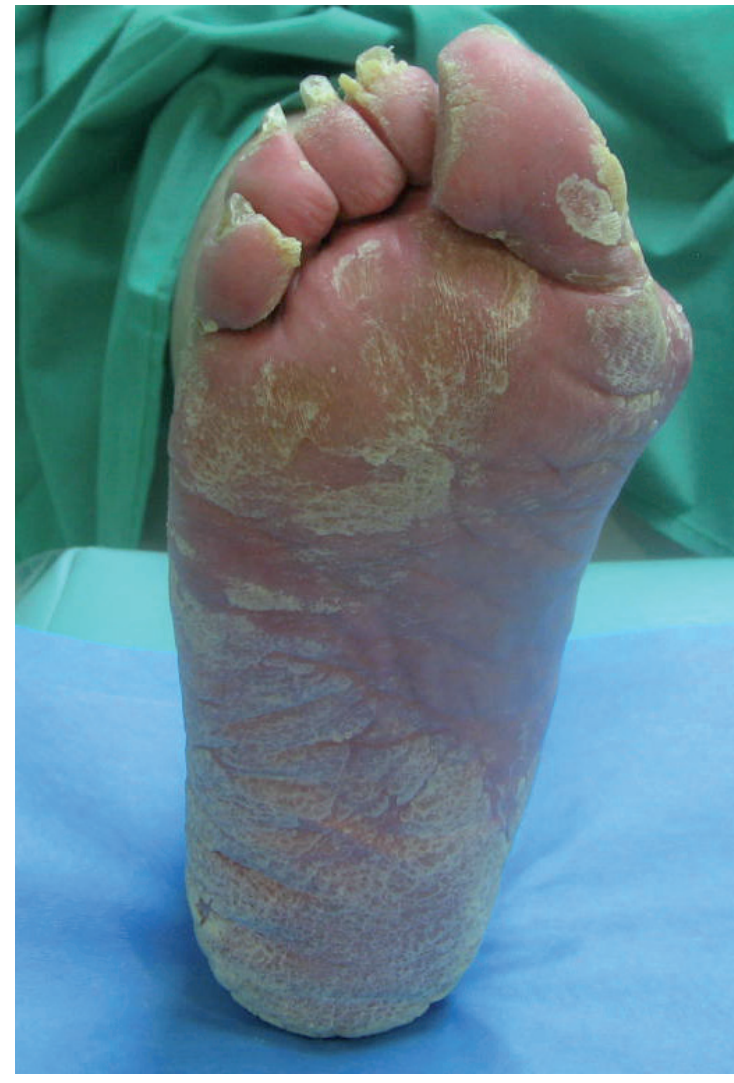

Figura 6. Psoriasis Eritrodérmica o Exfoliativa en el pie derecho.

\section{DIAGNÓSTICO}

Existen diferentes métodos para realizar el diagnóstico, pero el más utilizado, barato y práctico es realizar una correcta historia clínica acerca de los síntomas comunes (véase Tabla 3) y una exploración física prestando atención al cuero cabelludo y a las uñas siendo estos los criterios utilizados actualmente que se recomiendan en el Consenso Internacional de Psoriasis $^{21,26}$.

Además, para evaluar la gravedad de la enfermedad existen diferentes formas, una de las más habituales es medir el porcentaje de la Superficie Corporal Afectada (SCA) que se utiliza para cuantificar la gravedad (extensión) de la psoriasis y para ello se utiliza como referencia la palma de la mano, los dedos y el pulgar para valorar el nivel de afectación de la piel que presenta psoriasis, lo que representa

\section{Puntos Clave en la Entrevista Clínica}

Síntomas / Lesiones/ Diagnóstico

Antecedentes Familiares

Manifestaciones sistémicas

Factores Exacerbantes

Factores a mejorar

Respuesta de tratamientos anteriores

Variedad de opciones terapéuticas

Enfermedades crónicas a largo plazo

Desórdenes Psicológicos

Optimismo para el futuro

Soporte / Servicios Disponibles de la Fundación Nacional de Psoriasis

Tabla 3. Localización y signos clínicos, según Menter y Weinstein, 2008.

más o menos $1 \%$ de la superficie del cuerpo y que según el grado de afectación se divide la psoriasis en tres tipos (véase Tabla 4) ${ }^{27,28}$.

Esta herramienta de diagnóstico rápido permite al clínico identificar la gravedad de la enfermedad, siendo la fiabilidad interanalista variable y la fiabilidad intraobservador excelente. Además se puede considerar grave el grado de severidad, cuando la afectación involucra a las palmas de las manos y las plantas de los pies 29,30 .

\section{TRATAMIENTO}

Hay una amplia gama de modalidades disponibles para el tratamiento de la psoriasis a nivel y general y en el pie a nivel especial que están relacionados con el área de afectación, con los distintos factores que confluyen en la patogenia 


\section{Tipos de Psoriasis $\quad$ Porcentaje (\%)}

Psoriasis Leve hasta el 3\% del cuerpo, generalmente en placas aisladas en las rodillas, codos, cuero cabelludo, manos y pies. A menudo se puede controlar con tratamientos tópicos

Psoriasis Moderada entre un 3 - 10\% de la superficie del cuerpo. A menudo aparece en los brazos, piernas, torso, cuero cabelludo y otras áreas. Los agentes tópicos, fototerapia, medicamentos sistémicos, incluyendo las terapias biológicas, puede ser apropiadas.

Psoriasis Grave $\quad>10 \%$ del cuerpo. Puede ser extensa con placas, pústulas o eritrodermia. La fototerapia, los medicamentos sistémicos, incluidos los biológicos o una combinación de éstos, con o sin un agente tópico suelen ser necesarias para lograr resultados adecuados.

Tabla 4. Clasificación de los tipos de Psoriasis.

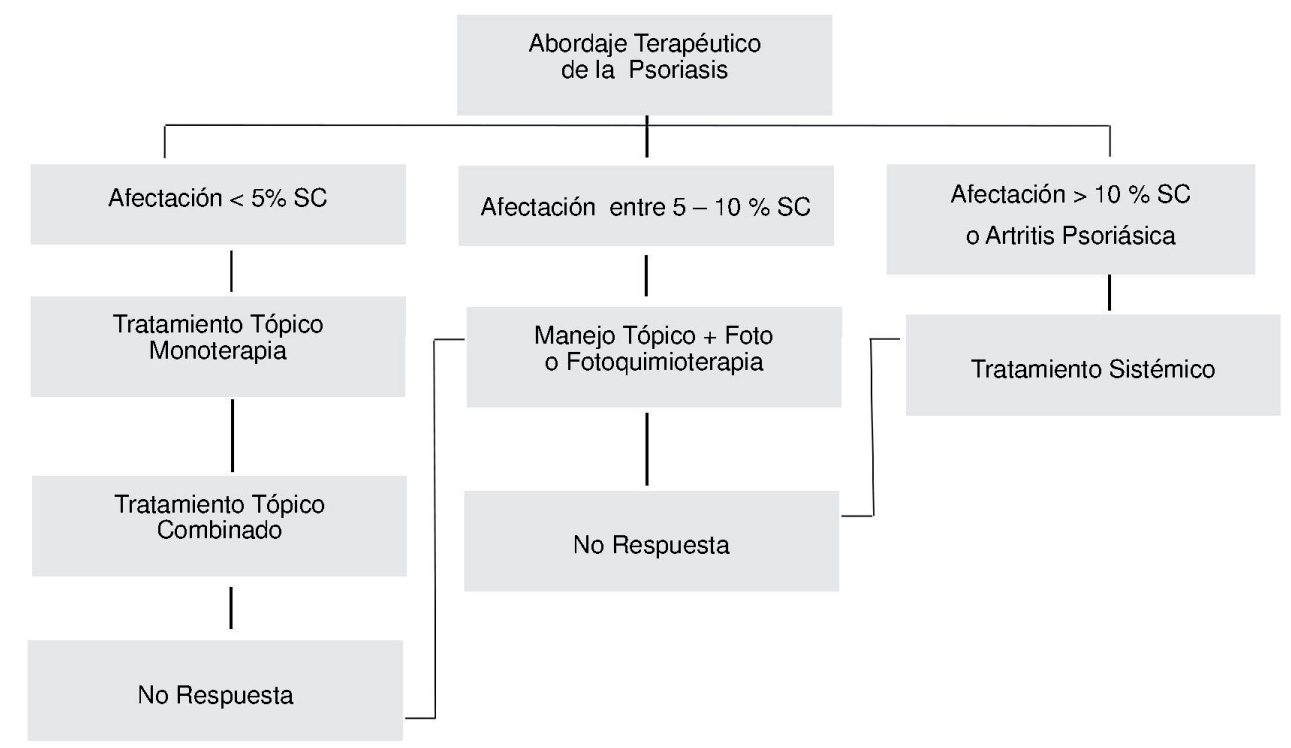

Figura 7. Algoritmo terapéutico del tratamiento de la Psoriasis, según Lebwohl y Van de Kerkhof, 2010.

y con la etiología multifactorial de la enfermedad, para lo cual el abordaje terapéutico está orientado al control de los pacientes y a evitar los efectos colaterales importantes que alteren la calidad de vida y la salud de las personas ${ }^{31}$.

Las guías de evidencia del tratamiento de la psoriasis basan la actuación terapéutica de primera línea en el manejo tópico de la enfermedad. Sin embargo, dada la variedad de expresión de la enfermedad, en ocasiones se hace insuficiente el manejo tópico y se debe recurrir al tratamiento sistémico, del cual existe una gran cantidad de opciones disponibles ${ }^{32}$ (véase Figura 7). 
El eje central de la primera línea de actuación se relaciona con la monoterapia que se utiliza a menudo como el abordaje inicial ya que un fármaco consigue limitar los efectos secundarios, disminuir los costes y mejorar la adherencia para el régimen de tratamiento. La desventaja a largo plazo con algunos agentes a dosis altas es que pueden incrementar la toxicidad en el organismo y que cese el efecto terapéutico.

Los distintos agentes que de manera habitual se pueden prescribir para el tratamiento de la psoriasis son:

1. Antralina o Ditranol (1,8-dihidroxiantrona): su uso habitual está definido para áreas recalcitrantes de psoriasis, se utilizan en distintos vehículos, formas de presentación y a veces en combinación con la fototerapia (UVB), siendo los efectos locales que genera los que se relacionan con la tinción e irritación cutánea y de ahí que su uso terapéutico en lesiones locales sea menos habitual ${ }^{33}$.

Las propiedades del ditranol son de tipo antiproliferativas y antinflamatorias al actuar sobre los queratinocitos y las células del infiltrado respectivamente, por lo que disminuye la hiperplasia epidérmica y la inflamación dérmica. Por otro lado, la antralina induce la transcripción del factor nuclear NF-kB. Dicho factor de transcripción está en relación con la expresión de otras citoquinas como IL-6, IL-8 y TNF- $\alpha$ hecho que permite explicar las propiedades irritantes de este producto ${ }^{34}$.

El tratamiento con antralina se inicia a bajas concentraciones $(0,05-0,1 \%)$ en vaselina o crema aplicándose sobre la placa de psoriasis una vez al día. Puede añadirse ácido salicílico al 0.5 - 1\% para prevenir la autooxidación. Progresivamente se va incrementando la concentración semanalmente según la tolerancia hasta alcanzar concentraciones de hasta el 5\%.

2. Corticoides Tópicos: es la terapia más prescrita y utilizada para el tratamiento de la psoriasis existen diferentes presentaciones disponibles como son las cremas, los emolientes, las lociones, las soluciones o los aerosoles ${ }^{35}$.

Las propiedades de los corticoides o corticoisteroides se relacionan con sus efectos
Clasificación Corticoesteroides Tópicos según potencia

CLASE I (Muy potentes)

Diflucortolona valerato $0,3 \%$

Clobetasol propionato $0,05 \%$

Halcinónido 0,1\%

Halobetasol propionato $0,05 \%$

\section{CLASE II (Potentes)}

Beclometasona dipropionato $0,025 \%$, crema, ungüento

Betametasona: dipropionato $0,05 \%$, ungüento

valerato $0,1 \%$, ungüento

Budesonida 0,05\%

Diflucortolona valerato $0,1 \%$

Metilprednisolona aceponato, $0,1 \%$

Mometasona furoato $0,1 \%$

Prednicarbato $0,25 \%$

\section{CLASE III (Potencia moderada)}

Beclometasona dipropionato $0,025 \%$ loción Betametasona dipropionata $0,05 \%$ loción

Hidrocortisona: aceponato $0,1 \%$ crema valerato $0,2 \%$

butirato $0,1 \%$

\section{CLASE IV (Potencia baja)}

Fluocortina butilo $0,75 \%$ crema

Hidrocortisona acetato $1 \%$

Metilprednisolona acetato $0,25 \%$

Tabla 5. Clasificación Potencia de los Corticoides, según Lázaro y Suarez, 2001. 


\section{Preparados de la Brea de Hulla}

Principio Activo

Brea e Hulla (Coaltar)

Brea de hulla

Brea de pino

Aceite de enebro

Aciete de cacahuete
Nombre Comercial Presentación

Alfitar ${ }^{\circledR}$

Tarmed $®$

Exorex ${ }^{\circledR}$

Piroxgel $\mathbb{R}$

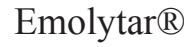

Polytar ${ }^{\circledR}$

Polytar plus ${ }^{\circledR}$

Solución $500 \mathrm{ml}$

Champú $250 \mathrm{ml}$
Solución $0,066 \% 50 \mathrm{~m}$

Solución $150 \mathrm{ml}$

Emulsión 100, $250 \mathrm{ml}$

Champú 6 mg/ml
Brea de hulla, Ácido salicílico

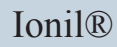
Champú $200 \mathrm{ml}$

Solución 150, $250 \mathrm{ml}$

Tabla 6. Preparados de la Brea de Hulla.

antiinflamatorios y antiproliferativos, siendo el eje de acción habitual en las lesiones psoriásicas y según Stoughton-Cornell, la clasificación se basa en la potencia y en la capacidad vasoconstrictora de cada uno de ellos, que a la vez está relacionada con su potencia antinflamatoria ${ }^{36}$.

Las potencias de los corticoides tópicos oscilan entre los de clase 4 (muy baja potencia), como la hidrocortisona al 1\%, disponible en las farmacias sin receta médica hasta los de clase 1 (superpotentes), como el propionato de clobetasol siendo los efectos secundarios los que se relacionan con la atrofia cutánea, las telangiectasias, las estrías, el acné, la erupción, el glaucoma, la supresión del eje hipotálamo-pituitarioadrenal, el retraso del crecimiento, entre otras (véase Tabla 5) 37,38. $^{\text {. }}$

3. Alquitranes (brea de hulla o coaltar): combinación de distintas sustancias obtenidas mediante condensación, algunas de ellas durante la combustión del carbón y de interés para la sensibilización de las radiaciones $\mathrm{UVA}^{39}$.
Las acciones del alquitrán se relacionan con sus constituyentes específicos como el benzopireno que son transformados en especies reactivas y que se unen a las macromoléculas celulares cruciales que tienen propiedades antiacantóticas, queratoplásticas y efectos atrofogénicos en humanos, al disminuir después de 6 - 8 semanas de tratamiento la hiperqueratosis y el estrato epidérmico.También tiene un efecto fotosensibilizante, vasoconstrictor, antibacteriano, antiparasitario, antifúngico y antipruriginoso $^{40}$.

La seguridad en el uso es una de sus virtudes al carecer de poder de toxicidad, aunque su papel en el tratamiento de la psoriasis, es subsidiario debido a los efectos secundarios que proporciona relacionados con la tinción que provoca en la piel y en la ropa $^{41,42}$ (véase Tabla 6).

4. Derivados de la Vitamina D (1,25 dihidroxicolecalciferol): de gran utilidad la administración de vitamina $\mathrm{D}$ oral en personas que presentan osteoporosis, pero no en personas que carecen de patología ósea, 
ya que producen hipercalcemia - hipercalciuria, siendo los análogos de la vitamina $\mathrm{D}$ tópicos con pocos efectos sistémicos los que generan efectividad en el tratamiento de la psoriasis.

Las propiedades de la vitamina D y los análogos como son el calcipotriol y el tacalcitol están relacionados con la actuación sobre receptores de la vitamina $\mathrm{D}$ que generan la modificación de la trascripción del material genético que inhibe a la multiplicación de los queratinocitos no diferenciados y estimula a los queratinocitos diferenciados a su queratinización. Además, la presencia de receptores de vitamina $\mathrm{D}$ en las células de Langerhans, en los queratinocitos, en los melanocitos, en los fibroblastos, en las células endoteliales, en los linfocitos activados, permiten que la actividad de estos productos abarque también la inmunoregulación de los principios activos.

El tratamiento con los análogos de la vitamina D de interés para la psoriasis son el Calcipotriol y el Tacalcitol, con un efecto mucho mejor que el de los corticosteroides siendo el efecto secundario más habitual el de la irritación de las lesiones y la hipercalcemia cuando su uso se incrementa por encima de las dosis permitidas 100 gramos semanales (véase Tabla 7) ${ }^{42}$.

5. Retinoides Tópicos: pertenecen a un grupo de compuestos formados por la vitamina A y por los análogos, tanto naturales como sintéticos y que se desarrollaron para evitar muchos de los efectos adversos sistémicos de los retinoides orales (etretinato). El tazaroteno es un retinoide tópico de uso en psoriais y actúa en procesos inflamatorios, en la proliferación e hipercornificación celular, regulando la expresión de diferentes genes y proteínas.

La presentación es en forma de gel al 0,1 y $0,05 \%$ (Zorac ${ }^{\circledR}$ gel), genera una mejora de un $50-70 \%$ de los casos de psoriasis, aunque tiene una alta capacidad de generar efectos secundarios como la irritación, la inflamación, el dolor punzante, el prurito, la descamación y el eritema, por ello, el uso debe ser combinado con corticoides ${ }^{43}$.

6. Inmunomoduladores tópicos: inhiben la síntesis de la calcineurina siendo los agen-

\section{Preparados que contienen análogos de vitamina D}

\begin{tabular}{|c|c|c|c|}
\hline & Posología & Dosis Máxima & $\begin{array}{l}\text { Duración Máxi- } \\
\text { ma }\end{array}$ \\
\hline Calcitriol (Silkis ${ }^{\circledR}$ pomada) & Cada $12 \mathrm{~h}$ & 30 g/día & 6 semanas \\
\hline $\begin{array}{l}\text { Calcipotriol } \\
\text { (Daivonex }{ }^{\circledR} \text { crema y solución) }\end{array}$ & $\begin{array}{l}\text { Cada } 12 \text { h si } \\
\text { remisión } \\
\text { cada } 24 \text { h) }\end{array}$ & $\begin{array}{l}100 \mathrm{~g} \text { (eq. } 5 \mathrm{mg} \\
\text { de calcipotriol) }\end{array}$ & \\
\hline Tacalcitol (Bonalfa ${ }^{\circledR}$ pomada) & Cada $24 \mathrm{~h}$ & Pomada 5 g/día & 12 semanas / año \\
\hline $\begin{array}{l}\text { Calcipotriol + betametasona } \\
\text { (Daivobet }{ }^{\circledR} \text { pomada, Xamiol }{ }^{\circledR} \text { gel) }\end{array}$ & Cada $24 \mathrm{~h}$ & $\begin{array}{l}15 \mathrm{~g} / \text { día y } 100 \mathrm{~g} / \\
\text { semana }\end{array}$ & 4 semanas \\
\hline
\end{tabular}

Tabla 7. Clasificación Análogos de a Vitamina D. 
tes de uso para la psoriaisis el tacrolimus $0,1 \%$ (Protopic $®$ ) y el o pimecrólimus $1 \%$ (Elidel ${ }^{\circledR}$, Rizan $\left.{ }^{\circledR}\right)$ que carecen de los efectos secundarios de los corticosteroides. La eficacia del tacrolimus es superior a la del pimecrolimús ${ }^{44}$.

Su mecanismo de acción es igual que los otros análogos, quizás influyendo más en la inhibición de alguna citoquina liberada por los queratinocitos.

A continuación, se recomienda la fototerapia y la foquimioterapia como el segundo paso en la terapia, siendo la radiación ultravioleta la que actúa como inmunosupresor local por sus efectos directos en las células de Langerhans y sus efectos indirectos en numerosas citoquinas y moléculas de adhesión, lo que lleva a un cambio en el fenotipo de las citoquinas, de Th1 a Th2, que ayuda a la mejoría de las lesiones ${ }^{44}$.

La fototerapia con UVB y las selectivas con longitudes de onda superiores a los $311 \mathrm{~nm}$ son de gran utilidad para la eliminación de los eritemas en la epidermis de las personas con psoriasis. Además la fotoquimioterapia o PUVA es una terapia que expone al paciente a radiación UVA tras dos horas de la ingestión oral del fármaco, o en algunos casos baño, de un psoraleno fotosensibilizante referente en cuanto a eficacia en el tratamiento de la psoriasis, si bien es también la variante de fototerapia asociada a un mayor número de efectos secundarios como los eritemas y la irritación de la piel a corto y a largo plazo. Esta circunstancia explica probablemente el creciente empleo del baño de PUVA, que reduciría en parte estos inconvenientes en los últimos años ${ }^{45}$. La utilización está enfocada al fracaso en los tratamientos tópicos o cuando las áreas de psoriasis son demasiado extensas $(>40 \%$ del área corporal). Ambos tipos de terapia exigen 2 ó 3 días por semana de aplicación durante 6 - 8 semanas con la consiguiente incomodidad por desplazamiento al centro clínico, donde se recomienda controlar la posible fotosensibilidad, la hepatotoxicidad por los psoralenos, el riesgo de cataratas $\mathrm{y}$, sobre todo, el peligro de desarrollar tumores cutáneos a largo plazo.
La tercera línea de abordaje terapéutico está relacionada con la administración sistémica en presencia de psoriasis extensas que no responden a otros tratamientos y relacionado con las formas eritrodérmicas, pustulosas e incapacitantes, donde es necesario tener en cuenta la necesidad de una asistencia periódica a las revisiones, el nivel educativo del paciente, los factores relacionados con la enfermedad, la extensión del compromiso cutáneo, la presencia de enfermedades concomitantes, etc,

La terapia inmonomudolora con metratexato semanal es de las más utilizadas en personas con psoriasis con una larga evolución de la enfermedad, donde la monitorización del paciente es esencial, ya que es un fármaco que tiene efectos adversos graves y de ahí la relevancia de realizar pruebas analíticas como hematocrito y pruebas de la función hepática y renal ${ }^{46}$.

Otro fármaco eficaz es ciclosporina oral de uso en el tratamiento intermitente y de corta duración, debido a la nefrotoxicidad y a causar hipertensión arterial que también necesita la monitorización y realizar pruebas complementarias que evalúen el estado general del paciente ${ }^{47}$.

La acitretina (Neotigasón ${ }^{\circledR} 10$ y $25 \mathrm{mg}$ ) es un retinoide análogo a la vitamina $\mathrm{D}$ y es una alternativa en aquellas personas que están inmunodeprimidos o que padecen psoriasis pustulosa, ya que mejora las lesiones y se puede combinar con la PUVA. Los controles periódicos son necesarios debido al efecto teratogénico que afecta hasta dos años después de su administración.

De interés en las diferentes opciones de tratamiento de la psoriaisis la relevancia de terapia combinada, que generalmente ayudan con unas dosis más bajas de agentes, a minimizar la toxicidad y a una mejorar eficacia terapéutica ${ }^{48}$ (véase Tabla 6).

\section{CONCLUSIONES}

La psoriasis en el pie es un trastorno frecuente con importantes consecuencias clínicas, siendo la presentación clínica variable, así como los 
Terapia Tópica

Antralina o Ditranol

\section{Fototerapia}

\section{Terapia Sistémica}

UVB Terapia Goeckerman Retinoides acitretina

(Neotigasón ${ }^{\circledR} 10$ y $25 \mathrm{mg}$ )

Terapia Inmunomoduladora con Metotrexato

UVB de Banda Ancha

Cremas de Corticosteroides, Lociones, Ungüentos, Geles, Espumas, Champús, Parches y Soluciones

Alquitranes

UVB de Banda Estrecha

Otra terapia modulación inmune
Derivados de la Vitamina D
PUVA
Ciclosporina

Retionoides Tópicos

Láser Excimer

Bloqueadores Biológicos del

TNF

Inmunomoduladores tópicos

Biológicos Anti-CD2

Bloqueadores Biológicos

IL12/23

Tabla 6. Opciones de tratamiento de la Psoriasis.

patrones de distribución, la severidad e impacto en las personas.

La variedad de opciones de tratamiento disponibles se deben enfocar en función de la gravedad y pueden ser medidas por medio de la SCA que facilita en la mayoría de los casos, la adaptación del tratamiento a cada paciente en la búsqueda de un resultado satisfactorio.

Sin embargo, además de la puntuación de la SCA y la localización, se deben tener en cuen- ta otros factores como la duración del efecto, los efectos secundarios, las complicaciones, el coste económico, así como la adherencia terapéutica del paciente, relacionada con la toma de decisiones en el abordaje terapéutico que debe utilizarse de forma individualizada y escalonada, teniendo en cuenta las indicaciones y las contraindicaciones específicas de cada medicamento.

\section{BIBLIOGRAFÍA}

1. Lebwohl M. Psoriasis. Lancet 2003; 361: 1197-1204.

2. Mrowietz U, Reich K. Psoriasis-New Insights Into Pathogenesis and Treatment. Dtsch Arztebl Int. 2009 January; 106(1-2): 11-19.

3. Kimball AB, Jacobson C, Weiss $\mathrm{S}$, Vreeland MG, Wu Y. The psychological burden of psoriasis. Am J Clin Dermatol 2005; 6 (6):383-92

4. Langley RG, Krueger GG, Griffiths CE. Psoriasis: epidemiology, clinical features, and quality of life. Ann Rheum Dis 2005, 64 (Suppl 2):ii18-23 
5. Augustin M, Reich K, Glaeske G, Schaefer I, Radtke M. Co-morbidity and age-related prevalence of psoriasis: analysis of health insurance data in Germany. Acta Derm Venereol 2010; 90: 147-151.

6. Tsai TF, Wang TS, Hung ST et al. Epidemiology and comorbidities of psoriasis patients in a national database in Taiwan. J Dermatol Sci 2011; 63: 40-46.

7. Farley E, Masrour S, McKey J, Menter A. Palmoplantar psoriasis: a phenotypical and clinical review with introduction of a new quality-of-life assessment tool. J Am Acad Dermatol 2009; 60(6):1024-31.

8. Marsland AM, Chalmers RJ, Hollis S et al. Interventions for chronic palmoplantar pustulosis. Cochrane Database Syst Rev 2006, 25;(1):CD001433.

9. Kurd SK, Troxel AB. Crits $\sim$ Christoph p. Gelfand $1 \mathrm{M}$. The risk of depression, anxiety and suicidality in patients with psoriasis: a population -based cohort study. Arch Dermaloi 2010; 14 6:89 1-5.

10. Yang YW, Keller JJ, Lin HC. Medical comorbidity associated with psoriasis in adults: a populationbased study. Br J Dermatol 2011; 165 (5): 1037-43

11. Dubertret L, Mrowietz U, Ranki A, van de Kerkhof PC, Chimenti S, Lotti T, Schäfer G. European patient perspectives on the impact of psoriasis: the EUROPSO patient membership survey. Br J Dermatol. 2006; 155(4):729-36.

12. National lnstitute for Health and Clinical Excellence. Psoriasis: The assessment and management of psoriasis. http://guidance.nice.org.uk/ CG/Wave23 /6.

13. Menter A, Korman NJ, Elmets CA, et al. Guidelines of care for the management of psoriasis and psoriatic arthritis: section 5. Guidelines of care for the treatment of psoriasis with phototherapy and photochemotherapy. J Am Acad Dermatol 2010; 62 (I): 114-35.

14. Zheng Y, Danilenko DM, Valdez P et al. Interleukin-22, a T(H)17 cytokine, mediates IL-23-induced dermal inflammation and acanthosis. Nature 2007; 445: 648-651.

15. Torti DC, Feldman SR. Interleukin-12, interleukin-23, and psoriasis: current prospects. J Am Acad Dermatol 2007; 57: 1059-1068.

16. Nestle FO, Kaplan DH, Barker J. Psoriasis. N Engl J Med. 2009; 361:496-509.

17. Reich $\mathrm{K}$. The concept of psoriasis as a systemic inflammation: implications for diseasemanagement. J Eur Acad Dermatol Venereol. 2012: 26 (2): 3-11.

18. Harder J, Schröder JM. Psoriatic scales: a promising source for the isolation of human skin-derived antimicrobial proteins. J Leukoc Biol 2005; 77: 476-86

19. Schön MP, Boehncke, WH. Psoriasis. N Engl J Med 2005; 352:1899-191.

20. Van de Kerkhof PCM. Clinical features. In: van de Kerkhof PCM, ed. Textbook of Psoriasis. Oxford, UK: Blackwell Science Ltd; 2003:3-29.

21. Papp K, Gulliver W, Lynde C, Poulin Y, Ashkenas J; Canadian Psoriasis Guidelines Committee. Canadian guidelines for the management of plaque psoriasis: overview. J Cutan Med Surg. 2011; 15(4):2109.

22. Langley RG, Saurat JH, Reich K. Recommendations for the treatment of nail psoriasis in patients with moderate to severe psoriasis: a dermatology expert group consensus JEADV, 2012, 26, 373-381.

23. Ballester MM, Senan R, Ferran M, Barcala F. Terapia tópica de la psoriasis en Atención Primaria. Sociedad Española de Medicina de Familia y Comunitaria, 2011.

24. Padín JM, López M, Martín S, García FJ. Psoriasis y afectación en el Pie. Podología Clínica 2005; monográfico: 6-16

25. Chalela JC, González C, Castro L. Guías de Manejo de Psoriasis.Consenso Colombiano. Asociación Colombiana de Dermatología y Cirugía Dermatológica, 2008.

26. Menter A, Weinstein GD. An overview of psoriasis. In: Koo YM, Lebwohl MG, Lee CS, eds. Therapy of Moderate-to-Severe Psoriasis. London: Informa Healthcare; 2008:1-26.

27. Van Voorhees AS, Feldman SR, Koo JY, Lebwohl MG, Menter A. The psoriasis and psoriatic arthritis pocket guide: treatment algorithms and management options. Portland (OR): National Psoriasis Foundation; 2009.

28. Long CC, Finlay AY, Averill RW. The rule of hand: 4 hand areas $=2$ FTU $=1$ g. Arch Dermatol. 1992; 128:1129- 30 .

29. Marks R, Barton SP, Shuttleworth D, Finlay AY. Assessment of disease progress in psoriasis. Arch Dermatol 1989; 125 (2):235-240.

30. Yune Y-M, Park SY, Oh HS, et al. Objective assessment of involved surface area in patients with psoriasis. Skin Res Technol 2003;9(4):339-342

31. Arango FA, Franco MF, Hoyos AM. Psoriasis: conceptos actuales en el tratamiento sistémico. Rev Asoc Colomb Dermatol 2011, 19: 35-42. 
32. Lebwohl M, van de Kerkhof P. Psoriasis. En: Lebwohl M, Heymann W, Jones J, Coulson I. Treatment of skin disease. Third edition.: Saunders-Elsevier; 2010, 626-36.

33. Manresa MJ, Moreno JA. Tratamientos tópicos de la psoriasis: actualización. Med Cutan Iber Lat Am 2005; 33(4):147-157.

34. Schimdt KN et al: Anti-psoriatic drug anthralin activates transcription factor NF-kB in murine keratinocytes. J Immunol 1996; 156:4514.

35. Asha G, Pardasani MD, Steven R, Feldman MD, Adele R, Clark PA.Treatment of Psoriasis: An Algorithm-Based Approach for Primary Care Physicians. Am Fam Physician. 2000 Feb 1;61(3):725-733

36. Cornell RC, Stoughton RB. Correlation of the vasoconstriction assay and clinical activity in psoriasis. Arch Dermatol 1985; 121:63-71.

37. Lázaro P, Suárez R. Actualización en el tratamiento de la psoriasis. Inf Ter Sist Nac Salud 2001; 25: 105-110.

38. Flores C, Labrador N, Sehtman A, Allevato MA. Coaltar. Dermatol 2007; 30: 184-189.

39. Georgi L, Novoa C, Lima A, Azulay R. Coaltar. Act Terap. Dermatol 1988; 11: 231-235.

40. Lebwohl M, Ali S. Treatment of Psoriasis. Part 2. Systemic therapies. J Am Acad Dermatol. 2001; 45:649-661.

41. Actualización en el tratamiento de la psoriasis. Infac 2011, 19 (3): 1-20.

42. González FJ, Montalvillo E, Sánchez P, Pérez M. Bol Rac Med 2004 12(4): 1-12.

43. Ribera M. Psoriasis Guía de Tratamientos. Badalona: Ediciones Medicas 2009, 22.

44. Menter A, Korman NJ, Elmets C, Feldman S, Gelfand J, Gordon K, et al. Guidelines of care for the management of psoriasis and psoriatic arthritis, Section 5. Guidelines of care for the treatment of psoriasis with phototherapy and photochemotherapy. J Am Acad Dermatol. 2010; 62:114

45. Carrascosa JM. Fototerapia y Fotoquimioterapia. Actas Dermosifiliogr 2004;95(5):259-84

46. Lebwohl M, Ali S. Treatment of Psoriasis. Part 2. Systemic therapies. J Am Acad Dermatol. 2001; 45:649-661.

47. Scottish Intercollegiate Guidelines Network. Diagnosis and management of psoriasis and psoriatic arthritis in adults. October 2010.

48. Utilización de medicamentos. Psoriasis: tratamiento. Bol Ter Andal. 1999;15(1). 